\title{
ASO Author Reflections: Consideration of a Modified Classification System for Gastric Cancer Based on Lauren Subtypes
}

\author{
Lin-Yong Zhao, MD, PhD and Jian-Kun Hu, MD, PhD, FRCS \\ Department of Gastrointestinal Surgery and Laboratory of Gastric Cancer, State Key Laboratory of Biotherapy, West \\ China Hospital, Sichuan University and Collaborative Innovation Center for Biotherapy, Chengdu, Sichuan Province, \\ China
}

\section{PAST}

Gastric cancer (GC), as one of the most common cancers and leading causes of cancer-related mortality globally, is a heterogeneous malignance, and the analysis of its molecular and clinical characteristics has been complicated by histological and etiological heterogeneity. Although the Lauren classification (LC) has been widely used as pivotal histologic subtypes to understand the GCs since it was proposed in 1965, Shah implemented with a modified Lauren classification (mLC) system on the basis of anatomic location and epidemiology, as well as histopathologic classification, and found that each subtype could be distinguished from each other by differentially expressed genes. ${ }^{1}$ However, no unanimous consensus has been reached so far on whether the $\mathrm{mLC}$ system is superior to LC system. This study aimed to clarify the clinical significance of the $\mathrm{mLC}$ and to compare it with the LC for GC patients from three institutions. ${ }^{2}$

ASO Author Reflections is a brief invited commentary on the article, "Superiority of Tumor Location-Modified Lauren Classification System for Gastric Cancer: A Multi-Institutional Validation Analysis.” Ann Surg Oncol. 2018;25:3257-3263.

(C) The Author(s) 2018

First Received: 15 October 2018;

Published Online: 29 October 2018

J.-K. Hu, MD, PhD, FRCS

e-mail: hujkwch@126.com

\section{PRESENT}

The modified Lauren classification (mLC) in our study was found to be closely related to the depth of tumor invasion $(\mathrm{T})$, lymph node metastasis $(\mathrm{N})$, and distant metastasis (M), implying that mLC was likely to be associated with biological behaviors and aggressive features. ${ }^{2}$ Besides, the $\mathrm{mLC}$ rather than the LC system was illustrated to be an independent prognostic factor, with a better prognostic discriminatory ability and predictive accuracy than LC system. Therefore, compared with the LC system, the mLC could be considered a more reliable prognostic factor and may improve the prognostic discriminatory ability and predictive accuracy for gastric cancer patients. We hope that there will be more specific findings and evidence provided in larger sample-sized studies about the mLC system, which evaluated its clinical utility and made a comparative analysis with the LC system.

\section{FUTURE}

Subtyping gastric cancer (GC) is not only to discriminate its survival prediction but to observe its heterogeneity in response to treatments, because that one-size-fits-all approach to GC therapy has no longer been utilized. Personized comprehensive multidiscipline therapy should be advocated widely. Apart from the histopathologic or anatomical classifications, such as WHO subtypes, Lauren classification, Borrmann subtypes, etc., molecular screening and optimized therapy according to the GC molecular classifications have to be taken into account, thus making the development of TCGA and ACRG subtypes for GCs, from the genomic and transcriptional level, respectively. ${ }^{3,4}$ All of these confirmed that GC is a heterogeneous disease both in molecular biology and histopathologic morphology. 
As a consequence, an integrated classification system will be preferred and utilized in the future. Therefore, further studies are needed to create a consensus with respect to different ways of classifications and their clinical relevance for widespread use.

DISCLOSURES The authors have no conflicts of interest to disclose.

OPEN ACCESS This article is distributed under the terms of the Creative Commons Attribution 4.0 International License (http://crea tivecommons.org/licenses/by/4.0/), which permits unrestricted use, distribution, and reproduction in any medium, provided you give appropriate credit to the original author(s) and the source, provide a link to the Creative Commons license, and indicate if changes were made.

\section{REFERENCES}

1. Shah MA, Khanin R, Tang L, et al. Molecular classification of gastric cancer: a new paradigm. Clin Cancer Res. 2011;17(9):2693-701.

2. Zhao L-Y, Wang J-J, Zhao Y-L, et al. Superiority of tumor location-modified Lauren Classification System for gastric cancer: a multi-institutional validation analysis. Ann Surg Oncol. 2018;25:3257-63.

3. The Cancer Genome Atlas Research Network. Comprehensive molecular characterization of gastric adenocarcinoma. Nature. 2014;513:202-9.

4. Cristescu R, Lee J, Nebozhyn M, et al. Molecular analysis of gastric cancer identifies subtypes associated with distinct clinical outcomes. Nat Med. 2015;21(5):449-56. 